OPEN ACCESS

Edited by:

Luca Bertolaccini,

European Institute of Oncology

(IEO), Italy

Reviewed by:

Savvas Lampridis,

Guy's and St Thomas' NHS

Foundation Trust, United Kingdom

Andrea De Vico,

Azienda UsI Teramo, Italy

${ }^{*}$ Correspondence:

Guowei Che

cheguowei_hx@aliyun.com

Specialty section:

This article was submitted to

Thoracic Surgery,

a section of the journa

Frontiers in Surgery

Received: 07 December 2021

Accepted: 03 January 2022

Published: 04 February 2022

Citation:

Shen C and Che G (2022) Case Report: Combined Small Cell Lung

Carcinoma With Pulmonary Adenocarcinoma.

Front. Surg. 9:830849.

doi: 10.3389/fsurg.2022.830849

\section{Case Report: Combined Small Cell Lung Carcinoma With Pulmonary Adenocarcinoma}

\author{
Cheng Shen and Guowei Che* \\ Department of Thoracic Surgery, West-China Hospital, Sichuan University, Chengdu, China
}

Background: Combined small cell lung carcinoma is defined as cancer tissues that mainly contain small cell lung cancer (SCLC) components with non-SCLC (NSCLC) histopathological types. The most common part of NSCLC is squamous cell carcinoma or large cell carcinoma. Combined SCLC (CSCLC) contains adenocarcinoma is extremely rare.

Case Presentation: We reported a case with surgically treated diagnosed as CSCLC with adenocarcinoma in an elderly and we summarized the clinical features of this disease. The patient has remained well for over 2 weeks after the treatment.

Conclusion: There are still few research reports on CSCLC. Since the survival time of patients with advanced CSCLC is shorter than that of simple SCLC, the recommended treatment for CSCLC is early detection and early surgery. In order to facilitate preoperative diagnosis and avoid misdiagnosis of such rare diseases, more cases need to be reported.

Keywords: combined small cell lung carcinoma, small cell lung carcinoma, surgery, prognosis, adenocarcinoma, case report

\section{INTRODUCTION}

The precise definition of combined small cell lung cancer is that the cancer foci are mainly small cell carcinoma with other types of non-small cell, including adenocarcinoma, squamous cell carcinoma, and large cell neuroendocrine carcinoma reported by the WHO (1). Only 13\% of primary lung tumors are small cell lung cancer (SCLC). SCLC was first described by Barnard in 1926 as oat celled sarcoma which is the first statement of combined SCLC (CSCLC) in the world. CSCLC is rare in clinical patients, accounting for $<1-3.2 \%$ of all $\operatorname{SCLC}(2,3)$. We report a case with surgically treated diagnosed as CSCLC with adenocarcinoma in an elderly and we summarized the clinical features of this disease.

\section{CASE REPORT}

A 73-year-old man was admitted to our hospital for a routine health check and found a lung nodule by chest X-ray. He had a history of smoking. He had smoked a pack of cigarettes a day for the past 20 years and has now quit smoking for 9 months. When inquiring about the condition at the outpatient clinic, he denied any symptoms such as chest pain, hoarseness, hemoptysis, cough, and difficulty breathing. Physical examination revealed normal breath sounds in both lung fields. There was no obvious abnormality in the laboratory test results. The results of his lung function test and cardiovascular examination were within the normal range. A contrast-enhanced CT scan revealed 

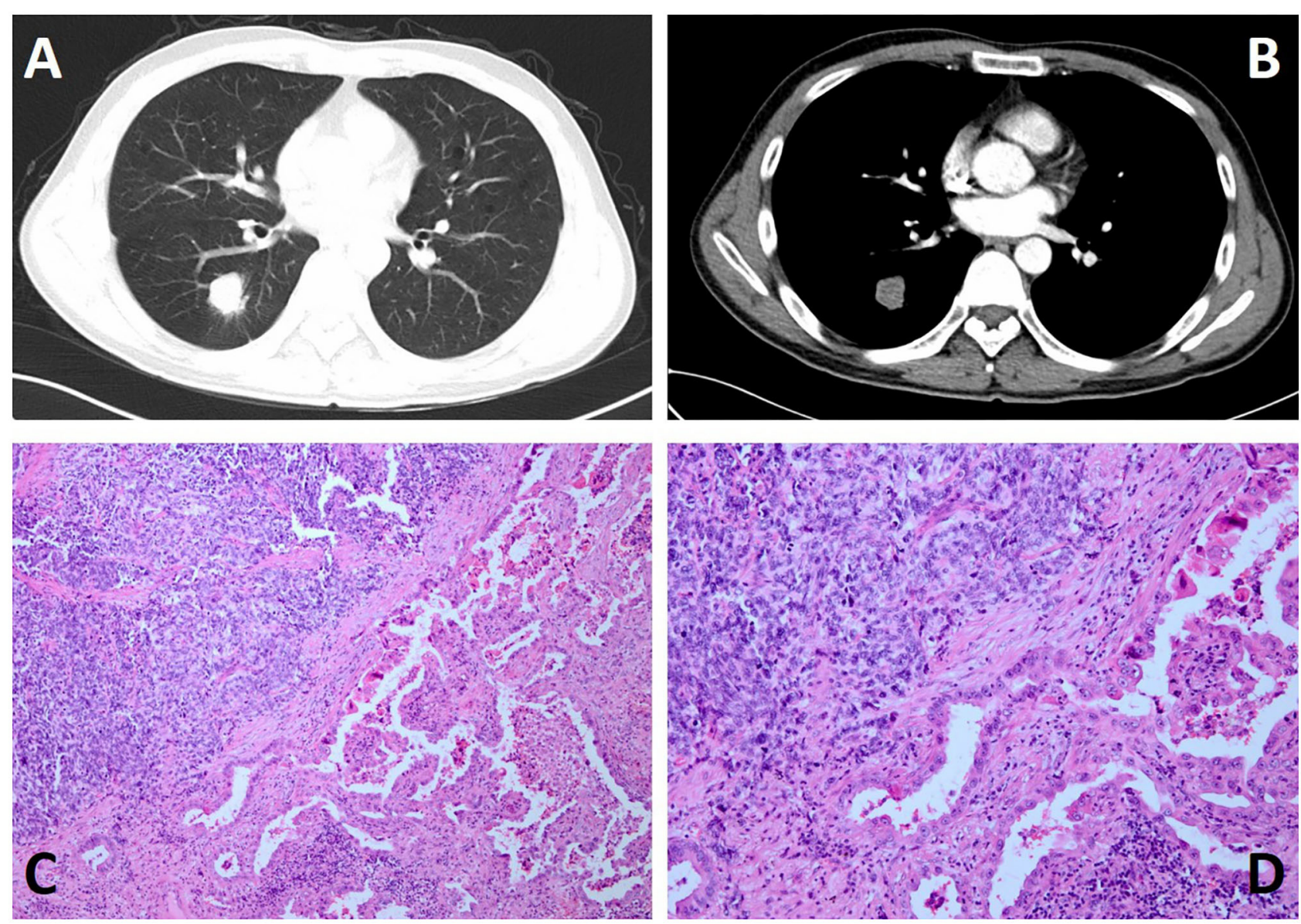

FIGURE 1 | Chest contrast-enhanced CT and histological features and of the case. (A,B) Contrast-enhanced CT scan showing a soft tissue, measuring $2.4 \mathrm{~cm} \times$ $3 \mathrm{~cm}$ in size, in the lower lobe of the right lung. (C,D) H\&E staining reveals the tumor consisted of dual disparate components, which were intermingled in a combined pattern with a definite boundary at low magnification $(\mathbf{C}: \times 100$ and $\mathbf{D}: \times 200)$.

a soft tissue mass measuring $2.4 \mathrm{~cm} \times 3 \mathrm{~cm}$ in the lower lobe of the right lung (Figures 1A,B). Bronchoscopy did not show evidence of pathology. Then, the patient was subjected to lobectomy by utilizing a three-port video-assisted thoracic surgery (VATS). During the operation, we did not find the lesion invaded the adjacent tissue structure. After we cut off the lesion, we used biopsy forceps to remove the tissue from the mass in the specimen plate for rapid freezing pathology, and the pathological diagnosis was small cell lung cancer combined with adenocarcinoma. The postoperative course was uneventful. The patient was followed up for 2 weeks without evidence of recurrence to date.

\section{DISCUSSION}

According to $2004 \mathrm{WHO}$ /International Association for the Study of Lung Cancer (IASLC) classification of lung and pleural tumors, CSCLC is defined as cancer tissues that mainly contain SCLC components with non-SCLC (NSCLC) histopathological types. The most common part of NSCLC is squamous cell carcinoma or large cell carcinoma $(4,5)$. CSCLC contains adenocarcinoma is extremely rare (6). Only 15 cases including our patient of pulmonary SCLC combined with adenocarcinoma have been reported in 7 studies. As summarized in our Table, the ratio of male and female was 4:1 and the mean age was 68 years (Table 1 ).

In our analysis, CSCLC is usually asymptomatic and may be diagnosed as an incidental finding and the majority of male patients are smokers, and it illustrates the correlation between CSCLC and smoking. Analyzing the collection cases in our single-center, the patient had no symptoms such as pain or cough, and he was discovered during routine health checkups. Because tumors are relatively rare, there is currently no definite radiological diagnostic standard for CSCLC. A chest radiograph is the most commonly used imaging method to evaluate lung mass, but it may not be able to distinguish CSCLC from pure SCLC.

Histopathologically, as seen in our case, the tumor lesion is composed of two completely different parts, which can be clearly defined under the microscope. In the SCLC part, the central component is mostly composed of small, uniform, poorly 
TABLE 1 | Clinicopathological features of the CSCLC.

\begin{tabular}{|c|c|c|c|c|c|c|c|c|c|}
\hline No & Years & Age & Gender & Smoking & Location & Size (cm) & Treatment & Follow up* & References \\
\hline 1 & 2021 & 79 & $\mathrm{M}$ & Yes & RLL & $3.2 \times 2.4 \times 2.0$ & VATS & Alive 2 weeks & Ours \\
\hline 2 & 2009 & 53 & $\mathrm{M}$ & Yes & RUL & 2.5 & thoracotomy+CT+RT & Dead 23 months & Wagner et al. (7) \\
\hline 3 & 2009 & 77 & M & Yes & LUL & 2 & thoracotomy+CT & Dead 16 months & Wagner et al. (7) \\
\hline 4 & 2009 & 72 & $\mathrm{~F}$ & None & LLL & 1.6 & thoracotomy+CT+RT & Dead 7 months & Wagner et al. (7) \\
\hline 5 & 2013 & 75 & $\mathrm{~F}$ & Not mention & RUL & Not mention & VATS & Alive 16.3 months & Babakoohi et al. (8) \\
\hline 6 & 2017 & 60 & $\mathrm{M}$ & Yes & LLL & $2.0 \times 2.0 \times 1.8$ & thoracotomy & Alive 12 months & Saito et al. (9) \\
\hline 7 & 2006 & 71 & $\mathrm{M}$ & Yes & LLL & 4 & thoracotomy+CT+RT & Alive 36 months & lezumi et al. (10) \\
\hline 8 & 2018 & 68 & $\mathrm{~F}$ & None & LLL & 5 & thoracotomy & Not mention & Bai et al. (11) \\
\hline 9 & 2003 & 68 & $\mathrm{M}$ & Not mention & RLL & 4 & thoracotomy+RT & Dead 1.5 months & Murase et al. (12) \\
\hline 10 & 2003 & 71 & $\mathrm{M}$ & Not mention & LUL & 2.5 & VATS & Alive 5 months & Murase et al. (12) \\
\hline 11 & 2003 & 73 & $\mathrm{M}$ & Not mention & RLL & 4 & thoracotomy & Dead 10 days & Murase et al. (12) \\
\hline 12 & 2007 & 74 & $\mathrm{M}$ & Yes & RLL & 3.1 & VATS & Not mention & Fukui et al. (13) \\
\hline 13 & 2007 & 62 & $\mathrm{~F}$ & None & LUL & 3.1 & VATS & Not mention & Fukui et al. (13) \\
\hline 14 & 2007 & 77 & $\mathrm{M}$ & Yes & LUL & 1.5 & pneumonectomy & Not mention & Fukui et al. (13) \\
\hline 15 & 2007 & 76 & $M$ & Yes & RUL & 2.8 & VATS & Not mention & Fukui et al. (13) \\
\hline
\end{tabular}

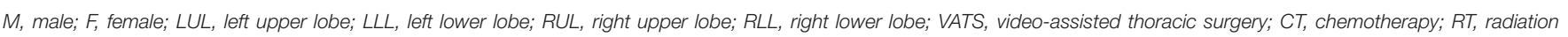
therapy; "survival times are post-resection.

differentiated necrotic cancers (Figures 1C,D), while the other half is considered to be adenocarcinoma with papillary and acinar features. Immunohistochemically, in the pulmonary tumor, TTF1 vimentin and pancytokeratin were strongly expressed in $75 \%$ of the small cell carcinoma cells, and $20 \%$ of the small cell carcinoma cells strongly expressed CD56, synaptophysin, and S100 protein $(10,11)$. As shown in Bai et al. (11) report, The components of SCLC are positive for synaptophysin (Syn), chromogranin A (CgA), and nerve cell adhesion molecule 1 (CD56), and have high proliferative activity by Ki-67 antigen immunostaining, while the adenocarcinoma area had low $\mathrm{Ki}-67$ proliferation activity and was negative to others.

In addition to the analysis of gross specimen morphology and pathology under a microscope, clonality analysis of each component is also useful in CSCLC. In the research of Wagner et al. (7), using loss of heterozygosity ( $\mathrm{LOH}$ ) analysis included 3 and $17 \mathrm{p}$ in the separate components of the lesion in each patient. In the study of Case 3, the alleles changing is inconsistent both in SCLC and NSCLC components. The alleles in SCLC are almost completely lost, while the number of the same alleles is greatly reduced in NSCLC. In case 2 in Table 1, LOH is demonstrated only in the SCLC component. The point mutations in the p53 gene and $\mathrm{LOH}$ of chromosome $3 \mathrm{p}$ in each component were examined and the results showed that the small cell carcinoma and squamous cell carcinoma components in CSCLC are clonerelated, but the adenocarcinoma component comes from a single clone and has no obvious correlation with small cell carcinoma (12).

Although small cell lung cancer is highly sensitive to chemotherapy and radiotherapy, most patients will eventually develop multiple organs metastasis of lung cancer cells. Surgical resection is only for patients with early-stage and no lymph node metastasis. The research suggests that the combination of etoposide and cisplatin is the traditional first-line treatment strategy for SCLC (9). In recent years, with the improvement of technology and detection methods, CSCLC has also been increasingly recognized by more surgeons. The role of surgery in the treatment of CSCLC is increasingly valued (6). For patients with no metastasis to the lymph node, lobectomy with systemic hilar and mediastinal lymphadenectomy are preferred (14). We can see from the Table 1 that the surgery was performed in all patients with video-assisted thoracic surgery (VATS) in six cases or lobectomy by standard thoracotomy in nine patients. Almost all patients with small cell lung cancer have a tendency to spread throughout the body. Therefore, combined chemotherapy and chest radiotherapy are the main treatments for this disease (15, 16). In Table 1, four patients underwent chemotherapy and three of them got radiation therapy. In case 7 , reported by Iezumi et al. (10), CSCLC patients received a combined chemotherapy regimen of cisplatin and irinotecan hydrochloride and received radiotherapy for metastasis and recurrence in the forearm. For CSCLC patients with EGFR mutations, use currently mature epidermal growth factor receptor (EGFR) tyrosine kinase inhibitors and other drug-related molecular targeted therapies to exert anti-tumor activity $(17,18)$.

Some researchers believe that pure SCLC and CSCLC have similar clinical manifestations and that the operation outcomes of both in a limited stage are similar. At present, the clinical characteristics and clinical data of these patients have been published $(8,13,19,20)$. In one study, 5-year survival of patients with CSCLC was only $15.9 \%$ (20). They collected the clinical data of CSCLC patients and pure SCLC patients and made detailed comparisons. The final data showed that the prognosis of CSCLC patients and SCLC patients were similar, and there was no statistically significant difference. However, the rate of surgery for CSCLC was much higher compared with pure SCLC. For those advanced patients who did not undergo surgery but received equivalent chemotherapy, the overall survival rate (OS) 
of CSCLC patients is lower than that of pure SCLC patients (13). Hage et al. (19) also reported that surgical resection in selected patients with pretreatment clinical stage I combined and pure SCLC can be curative or offer long-term survival. However, The OS of patients with pure SCLC is significantly lower than that of patients with CSCLC in Babakoohi et al. (8) research. In their study, the 5-year survival rate of CSCLC patients with stage I or stage II was very good, almost reaching 100\%, which is in contrast with the previous study by Hage et al. There are several limitations in this report. Firstly, the short-term followup is not enough to fully explain the prognosis in our patient. Secondly, the number of studies included and the simple scale were relatively small.

In short, there are still few research reports on CSCLC. Since the survival time of patients with advanced CSCLC is shorter than that of simple SCLC, the recommended treatment for CSCLC is early detection and early surgery. Surgical resection can only be performed in a small number of patients with conditions, whose can be cured or can obtain long-term survival from preoperative evaluation. In order to facilitate preoperative diagnosis and avoid misdiagnosis of such rare diseases, more cases need to be reported.

\section{DATA AVAILABILITY STATEMENT}

The original contributions presented in the study are included in the article/supplementary material, further inquiries can be directed to the corresponding author/s.

\section{REFERENCES}

1. Zhao X, McCutcheon JN, Kallakury B, et al. Combined small cell carcinoma of the lung: is it a single entity? J Thorac Oncol. (2018) 13:23745. doi: 10.1016/j.jtho.2017.10.010

2. Qin J, Lu H. Combined small-cell lung carcinoma. Onco Targets Ther. (2018) 11:3505-11. doi: 10.2147/OTT.S159057

3. Ebisu Y, Ishida M, Saito T, Murakawa T, Uemura Y, Tsuta K. Combined small cell carcinoma with giant cell carcinoma component of the lung: a case successfully diagnosed by computed tomography-guided fine-needle aspiration cytology. Oncol Lett. (2018) 15:1907-11. doi: 10.3892/ol.2017.7448

4. Asahina M, Fukumura Y, Mamat O, et al. A case of combined small cell lung carcinoma with unique morphology: Investigation of tumorigenesis. Pathol Int. (2018) 68:618-23. doi: 10.1111/pin.12726

5. Purkait S, Jain D, Madan K, Mathur S, Iyer VK. Combined small cell carcinoma of the lung: a case diagnosed on bronchoscopic wash cytology and bronchial biopsy. Cytopathology. (2015) 26:197-9. doi: 10.1111/cyt.12145

6. Wallace AS, Arya M, Frazier SR, Westgate S, Wang Z, Doll D. Combined small-cell lung carcinoma: an institutional experience. Thorac Cancer. (2014) 5:57-62. doi: 10.1111/1759-7714.12059

7. Wagner PL, Kitabayashi N, Chen YT, Saqi A. Combined small cell lung carcinomas: genotypic and immunophenotypic analysis of the separate morphologic components. Am J Clin Pathol. (2009) 131:37682. doi: 10.1309/AJCPYNPFL56POZQY

8. Babakoohi S, Fu P, Yang M, Linden PA, Dowlati A. Combined SCLC clinical and pathologic characteristics. Clin Lung Cancer. (2013) 14:1139. doi: 10.1016/j.cllc.2012.07.002

9. Saito T, Tsuta K, Fukumoto KJ, et al. Combined small cell lung carcinoma and giant cell carcinoma: a case report. Surg Case Rep. (2017) 3:52. doi: 10.1186/s40792-017-0328-9

\section{ETHICS STATEMENT}

Written informed consent was obtained from the individual(s) for the publication of any potentially identifiable images or data included in this article.

\section{AUTHOR CONTRIBUTIONS}

CS was involved in drafting the manuscript. GC designed and revised the manuscript. All authors have read and approved the final manuscript.

\section{FUNDING}

The Science and Technology Project of the Health Planning Committee of Sichuan (No. 19PJ242), Sichuan Province Science and Technology Support Program (No. 2020JDKP0023), and Chengdu science and technology Support Program (No. 2019YFYF-00090-SN).

\section{ACKNOWLEDGMENTS}

We greatly appreciate the assistance of the staff of the Department of Thoracic Surgery, West-China Hospital, Sichuan University, and thank them for their efforts.

10. Iezumi K, Masunaga A, Kadofuku T, et al. Combined small cell carcinoma with pulmonary blastoma and adenocarcinoma: case report and clonality analysis. Pathol Res Pract. (2006) 202:895-9. doi: 10.1016/j.prp.2006. 09.002

11. Bai Q, Li Y, Zhang X, Zhu X, Zhou X, A. Case of primary pulmonary combined small cell carcinoma with adenocarcinoma harboring the same KLC1-ALK fusion in both histologic components. J Thorac Oncol. (2018) 13:e197-9. doi: 10.1016/j.jtho.2018.05.018

12. Murase $\mathrm{T}$, Takino $\mathrm{H}$, Shimizu $\mathrm{S}$, et al. Clonality analysis of different histological components in combined small cell and non-small cell carcinoma of the lung. Hum Pathol. (2003) 34:1178-84. doi: 10.1053/j.humpath. 2003.05.001

13. Fukui T, Tsuta K, Furuta K, et al. Epidermal growth factor receptor mutation status and clinicopathological features of combined small cell carcinoma with adenocarcinoma of the lung. Cancer Sci. (2007) 98:17149. doi: 10.1111/j.1349-7006.2007.00600.x

14. Fujiwara $M$, Horiguchi $M$, Inage $Y$, Horiguchi $H$, Satoh $H$, Kamma H. Combined small cell carcinoma in the peripheral lung: importance of appropriate sampling. Acta Cytol. (2005) 49: 575-8.

15. Zhou K, Zhao S, Guo W, Ding L. Efficacy and safety of erlotinib combined with bevacizumab in the treatment of non-small cell lung cancer: a systematic review and meta-analysis. Medicine (Baltimore). (2020) 99:e18771. doi: 10.1097/MD.0000000000018771

16. Liu M, Wang $\mathrm{X}$, Li $\mathrm{H}, \mathrm{Xu} \mathrm{L}$, Jing L, Jiang $\mathrm{P}$, et al. The effect of apatinib combined with chemotherapy or targeted therapy on non-small cell lung cancer in vitro and vivo. Thorac Cancer. (2019) 10:186878. doi: 10.1111/1759-7714.13162

17. Tanaka T, Ishiki H, Kubo E, Yokota S, Shimizu M, Kiuchi D, et al. Is gefitinib combined with platinum-doublet chemotherapy a counterpart to osimertinib 
monotherapy in advanced EGFR-mutated non-small-cell lung cancer in the first-line setting? J Clin Oncol. (2020) 38:JCO1902509. doi: 10.1200/JCO. 19.02509

18. Li X, Liu M, Zhang H, Liu H, Chen J. Clinical study of apatinib combined with EGFR-TKI in the treatment of chronic progression after EGFR-TKI treatment in non-small cell lung cancer (ChiCTR1800019185). Thorac Cancer. (2020). doi: 10.1111/1759-7714. 13303

19. Hage R, Elbers JR, Brutel de. la Riviere A, van den Bosch JM. Surgery for combined type small cell lung carcinoma. Thorax. (1998) 53:450-3. doi: 10.1136/thx.53. 6.450

20. Luo J, Wu FY Li AW, Zheng D, Liu JM. Comparison of vinorelbine, ifosfamide and cisplatin (NIP) and etoposide and cisplatin (EP) for treatment of advanced combined small cell lung cancer (cSCLC) patients: a retrospective study. Asian Pac J Cancer Prev. (2012) 13:4703-6. doi: 10.7314/APJCP.2012.13. 9.4703
Conflict of Interest: The authors declare that the research was conducted in the absence of any commercial or financial relationships that could be construed as a potential conflict of interest.

Publisher's Note: All claims expressed in this article are solely those of the authors and do not necessarily represent those of their affiliated organizations, or those of the publisher, the editors and the reviewers. Any product that may be evaluated in this article, or claim that may be made by its manufacturer, is not guaranteed or endorsed by the publisher.

Copyright $\odot 2022$ Shen and Che. This is an open-access article distributed under the terms of the Creative Commons Attribution License (CC BY). The use, distribution or reproduction in other forums is permitted, provided the original author(s) and the copyright owner(s) are credited and that the original publication in this journal is cited, in accordance with accepted academic practice. No use, distribution or reproduction is permitted which does not comply with these terms. 